\title{
Aufnahme ausgewählter Metalle in Kompartimente von Solanaceen
}

D. Riße, H. Lahl* und B. Unterhalt

Institut für Pharmazeutische Chemie der Westfälischen Wilhelms-Universität Münster, Hittorfstr. 58-62, D-48149 Münster, Bundesrepublik Deutschland

\section{Uptake of selected metals into the compartments of Solanaceae}

The Uptake of several metals into the compartments of Atropa belladonna, Datura stramonium, and Hyoscyamus niger is measured by ICP-AES. In the roots the series $\mathrm{Al}<\mathrm{Ba}<\mathrm{Cr}<\mathrm{Cu}$ $<\mathrm{Zn}<\mathrm{Sr}$ is observed.

(Keywords: Solanaceae, uptake of metals by the compartments of plants, ICP-AES)

\section{Einleitung}

Als Folge des technischen Fortschritts werden verstärkt Metalle in die Umwelt freigesetzt, wobei die Atmosphäre die größte Bedeutung als Transportmedium hat. Metalle und deren Verbindungen gelangen meistens in Form von Aerosolen oder mit dem Niederschlagswasser in den Boden, aus dem sie von den Pflanzen aufgenommen werden. Ihre Anreicherung in der Nahrungskette, deren letztes Glied der Mensch ist, fordert zunehmende Aufmerksamkeit. So ist es das Ziel dieser Arbeit, quantitative Aussagen zur Aufnahme von $\mathrm{Al}, \mathrm{Cr}, \mathrm{Cu}, \mathrm{Zn}, \mathrm{Sr}$ und $\mathrm{Ba}$ aus dem Boden durch arzneilich relevante Solanaceen zu machen und die Verteilung der Elemente zu bestimmen. Sämtliche Pflanzen wurden aus Samen angezogen, deren Stammpflanzen in Vorversuchen auf den Parzellen des Arzneipflanzengartens gewachsen waren [1].

\section{Untersuchungen und Ergebnisse}

Begonnen wurde mit ICP-AES-Messungen des Boden-Referenzmaterials und der Bodenproben. Die in Tab. 1 aufgeführten Werte werden zur Berechnung von Transferquotienten herangezogen (Tab. 2). Diese beschreiben den Übergang von Metallen aus dem Boden in die Wurzeln [(W/Bo)-Transferquotient] bzw. die Beweglichkeit der Metalle innerhalb der Pflanzen: Blätter/Wurzel(Bl/W)-Transferquotient. 
Tab.1: Elementgehalt an $\mathrm{Al}, \mathrm{Cr}, \mathrm{Cu}, \mathrm{Zn}, \mathrm{Sr}$ und $\mathrm{Ba}$ im Referenzmaterial BCR No. 142, Light sandy soil und im Boden der Versuchsfelder, analysiert mit Hilfe der ICP-AES nach Druckaufschluß, $n=3$.

\begin{tabular}{cccccccc}
\hline & & $\mathrm{Al}$ & $\mathrm{Cr}$ & $\mathrm{Cu}$ & $\mathrm{Zn}$ & $\mathrm{Sr}$ & $\mathrm{Ba}$ \\
\hline BCR & Analyse & $44420 \pm$ & $93,1 \pm 4,2$ & $26,7 \pm 2,2$ & $94,3 \pm 4,2$ & $155 \pm$ & $510 \pm$ \\
No.142 & & 1570 & & & & 7 & 25 \\
BCR & Zertifiziert & $48700^{*}$ & $66-97^{*}$ & $27,5 \pm 0,6$ & $92,4 \pm 4,4$ & $164^{*}$ & $472^{*}$ \\
$\begin{array}{c}\text { No.142 } \\
\text { Boden }\end{array}$ & Analyse & $19920 \pm$ & $219 \pm$ & $26,3 \pm 4,2$ & $64,1 \pm 9,4$ & $97,7 \pm 4,0$ & $368 \pm$ \\
& & 1040 & 22 & & & & 79 \\
\hline
\end{tabular}

Angaben in $\mathrm{mg} / \mathrm{kg}$ Trockenmasse, ${ }^{*}$ Informationswert des BCR

\section{Aluminium- und Chrom-Verteilung}

Die gemessene Verteilung von Aluminium und Chrom in Kompartimenten von Datura stramonium, Hyoscyamus niger und Atropa belladonna im Verlauf der Vegetationsperiode ist in Abb. 1 dargestellt.

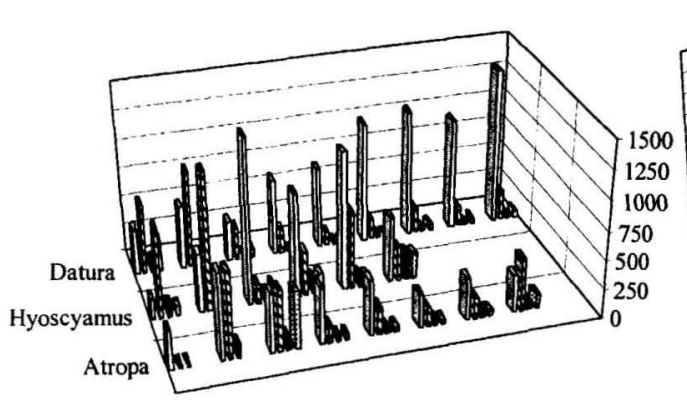

Aluminium

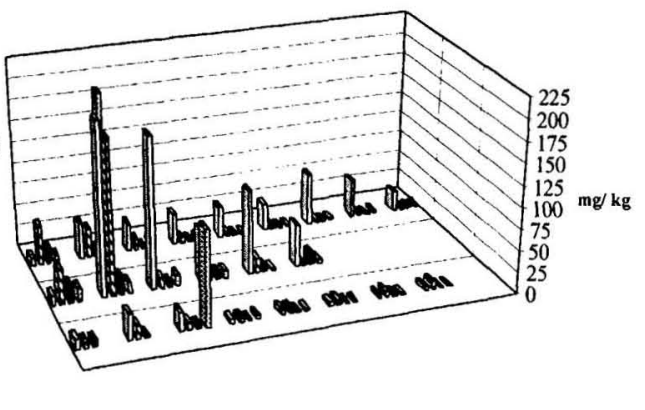

Chrom

Abb. 1: Aluminium- und Chrom-Konzentrationen in den Kompartimenten Wurzeln, Blätter, Stengel und Blüten/Früchte (Reihenfolge innerhalb einer Ernte von links nach rechts) von Datura stramonium (9 Ernten) und Hyoscyamus niger (6 Ernten) sowie Atropa belladonna im Verlauf von 2 Jahren (3 Ernten im ersten, 5 Ernten im zweiten Jahr); Messwerte [1].

Die höchsten Aluminium- und Chrom-Konzentrationen werden für Datura bei den ersten beiden Ernten in den Blättern gefunden; ab der dritten Ernte weisen die Wurzeln die höchsten Gehalte auf. In den Blättern sind die Konzentrationen höher als in der Blüten/Früchte-Fraktion und hier meist doppelt so hoch wie in den Stengeln. $50 \%$ des gesamten in der Pflanze enthaltenen Aluminiums ist gegen Versuchsende in den Wurzeln lokalisiert, deren Anteil an der Gesamttrockenmasse aber nur noch etwa $5 \%$ beträgt. Für Chrom ist die mit $193 \mathrm{mg} / \mathrm{kg} \mathrm{sehr}$ hohe Konzentration in der Blüten/Früchte-Fraktion der zweiten Ernte auffällig.Bei Hyoscyamus werden mit Ausnahme der ersten beiden Ernten die höchsten Aluminium- und ChromKonzentrationen ebenfalls in den Wurzeln gefunden, doch sind die Unterschiede zu den übrigen Kompartimenten weniger stark ausgeprägt als bei Datura. 
Atropa zeigt sich uneinheitlich. Für Aluminium werden bei der Mehrzahl der Proben die höchsten Konzentrationen in den Wurzeln gemessen; bei der 2., 3. und 8. Ernte jedoch weisen die Blätter höhere Gehalte auf. Zwischen 68 und $81 \%$ des Aluminiums findet sich in den Wurzeln. Die Konzentrationen der Organfraktionen von Atropa sind vergleichbar mit denen von Datura, die in den Wurzeln liegen jedoch um etwa die Hälfte niedriger. Für Chrom zeigt sich im ersten Versuchsjahr noch z.T. die typische Verteilung auf die Organfraktionen, im zweiten Versuchsjahr jedoch werden gleichhohe bis leicht erhöhte Konzentrationen in den Blättern gefunden. Transferquotienten sind Tab. 2 zu entnehmen [2].

Tab. 2: Wurzel/Boden (W/Bo)- und Blätter/Wurzel (Bl/W)-Transferquotienten furr Aluminium und Chrom bei Datura stramonium, Hyoscyamus niger und Atropa belladonna.

\begin{tabular}{cccccc|ccccccc}
\hline \multicolumn{9}{c|}{ Aluminium } & \multicolumn{5}{c}{ Chrom } \\
\hline Ernte & \multicolumn{2}{c}{$\begin{array}{c}\text { Datura } \\
\text { stramonium }\end{array}$} & \multicolumn{2}{c|}{$\begin{array}{c}\text { Hyoscyamus } \\
\text { niger }\end{array}$} & \multicolumn{2}{c}{$\begin{array}{c}\text { Atropa } \\
\text { belladonna* }\end{array}$} & \multicolumn{2}{c}{$\begin{array}{c}\text { Datura } \\
\text { stramonium }\end{array}$} & \multicolumn{2}{c}{$\begin{array}{c}\text { Hyoscyamus } \\
\text { niger }\end{array}$} & \multicolumn{2}{c}{$\begin{array}{c}\text { Atropa } \\
\text { belladonna* }\end{array}$} \\
& W/Bo & Bl/W & W/Bo & Bl/W & W/Bo & Bl/W & W/Bo & Bl/W & W/Bo & B1/W & W/Bo & B1/W \\
\hline 1 & 0,020 & $\mathbf{1 , 5 4 4}$ & 0,009 & $\mathbf{4 , 2 4 7}$ & 0,017 & 0,090 & 0,041 & $\mathbf{5 , 1 4 9}$ & $\mathbf{0 , 0 5 3}$ & $\mathbf{3 , 5 5 9}$ & 0,057 & 0,551 \\
2 & 0,027 & $\mathbf{1 , 5 5 1}$ & 0,026 & $\mathbf{2 , 3 2 6}$ & 0,038 & 1,009 & 0,195 & 0,788 & 0,910 & $\mathbf{0 , 8 9 6}$ & 0,139 & 0,484 \\
3 & 0,017 & 0,793 & 0,072 & 0,116 & 0,026 & 1,035 & 0,150 & 0,354 & 0,802 & 0,067 & 0,100 & 0,390 \\
4 & 0,033 & 0,311 & 0,045 & 0,429 & 0,024 & 0,313 & 0,164 & 0,168 & 0,274 & 0,186 & 0,022 & 0,969 \\
5 & 0,035 & 0,169 & 0,060 & 0,566 & 0,024 & 0,484 & 0,169 & 0,095 & 0,446 & 0,165 & 0,030 & 0,908 \\
6 & 0,053 & 0,163 & 0,029 & 0,519 & $\mathbf{0 , 0 1 5}$ & 0,567 & 0,144 & 0,104 & 0,230 & 0,056 & 0,021 & 1,253 \\
7 & 0,055 & 0,204 & - & - & 0,018 & 0,503 & 0,273 & 0,068 & - & - & 0,019 & 1,602 \\
8 & 0,049 & 0,179 & - & - & 0,016 & 1,433 & 0,203 & 0,053 & - & - & 0,025 & 1,570 \\
9 & 0,068 & 0,195 & - & - & - & - & 0,190 & 0,143 & - & - & - & - \\
\hline
\end{tabular}

*1.Versuchsjahr 1997: Emte 1 bis 3; 2.Versuchsjahr 1998: Ernte 4 bis 8; Messwerte [1]

\section{Kupfer- und Zink-Verteilung}

Die Verteilung von Kupfer und Zink in Datura stramonium, Hyoscyamus niger und Atropa belladonna im Verlauf der Vegetationsperiode ist Abb. 2 zu entnehmen.

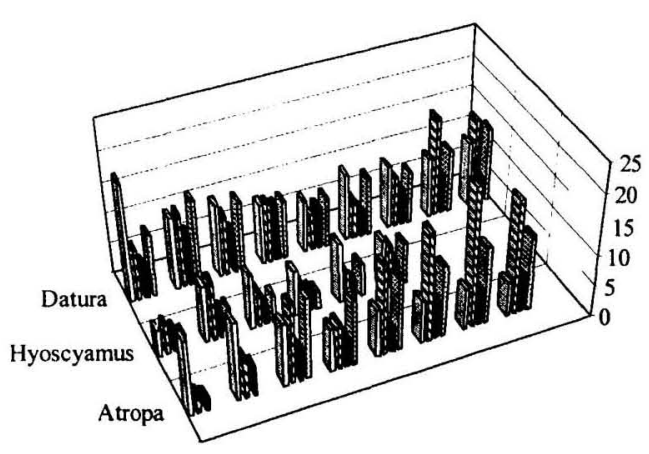

Kupfer

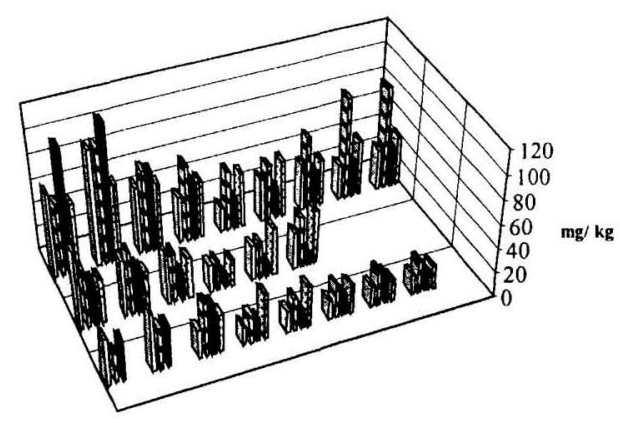

Zink

Abb. 2: Kupfer- und Zink-Konzentrationen in den Kompartimenten Wurzeln, Blätter, Stengel und Blüten/Früchte (Reihenfolge innerhalb einer Ernte von links nach rechts) von Datura stramonium (9 Ernten) und Hyoscyamus niger ( 6 Ernten) sowie Atropa belladonna im Verlauf von 2 Jahren (3 Ernten im ersten, 5 Ernten im zweiten Jahr); Messwerte [1]. 
Kupfer und Zink zeigen in Datura eine ganz andere Verteilung als Aluminium und Chrom. Die Unterschiede zwischen den einzelnen Organfraktionen sind gering, was auf eine gute Beweglichkeit in der Pflanze schließen läßt. Bei den ersten beiden Ernten ist auffällig, dass die Stengel sehr hohe Zink-Konzentrationen aufweisen. Ab der dritten Ernte enthalten die Blätter den höchsten Anteil an Kupfer und Zink, gefolgt von den Blüten/Früchten.

In Hyoscyamus sind die Unterschiede zwischen den Fraktionen generell schwach ausgeprägt. $\mathrm{Ab}$ der dritten Ernte weisen die Wurzeln die höheren Kupfer-Werte auf, während Zink vermehrt in den Blättern anzutreffen ist.

Bei Atropa werden im ersten Jahr die höchsten Kupfer- und Zink-Konzentrationen, mit Ausnahme der dritten Ernte, in den Wurzeln gefunden. Im zweiten Jahr weisen die Blätter die höchsten Kupfer-Konzentrationen auf, während Zink sich in Blätter und Blüten/Früchte verlagert. Transferquotienten sind Tab. $3 \mathrm{zu}$ entnehmen.

Tab. 3: Wurzel/Boden (W/Bo)- und Blätter/Wurzel (Bl/W)-Transferquotienten fur Kupfer und Zink bei Datura stramonium, Hyoscyamus niger und Atropa belladonna.

\begin{tabular}{|c|c|c|c|c|c|c|c|c|c|c|c|c|}
\hline \multirow{3}{*}{ Ernte } & \multicolumn{6}{|c|}{ Kupfer } & \multicolumn{6}{|c|}{ Zink } \\
\hline & \multicolumn{2}{|c|}{$\begin{array}{c}\text { Datura } \\
\text { stramonium }\end{array}$} & \multicolumn{2}{|c|}{$\begin{array}{l}\text { Hyoscyamus } \\
\text { niger }\end{array}$} & \multicolumn{2}{|c|}{$\begin{array}{c}\text { Atropa } \\
\text { belladonna* }\end{array}$} & \multicolumn{2}{|c|}{$\begin{array}{c}\text { Datura } \\
\text { stramonium }\end{array}$} & \multicolumn{2}{|c|}{$\begin{array}{c}\text { Hyoscyamus } \\
\text { niger }\end{array}$} & \multicolumn{2}{|c|}{$\begin{array}{c}\text { Atropa } \\
\text { belladonna* }\end{array}$} \\
\hline & $\mathrm{W} / \mathrm{Bo}$ & $\mathrm{Bl} / \mathrm{W}$ & $\mathrm{W} / \mathrm{Bo}$ & $\mathrm{Bl} / \mathrm{W}$ & $\mathrm{W} / \mathrm{Bo}$ & $\mathrm{B} 1 / \mathrm{W}$ & $\mathrm{W} / \mathrm{Bo}$ & $\mathrm{B} 1 / \mathrm{W}$ & $\mathrm{W} / \mathrm{Bo}$ & $\mathrm{B} 1 / \mathrm{W}$ & $\mathrm{W} / \mathrm{Bo}$ & $\mathrm{B} 1 / \mathrm{W}$ \\
\hline 1 & 0,738 & 0,423 & 0,162 & 1,127 & 0,464 & & 1,086 & 0,858 & 0,986 & 0,720 & 0,612 & 0,635 \\
\hline 2 & & & 0,3 & 1,0 & & & & & 0,730 & & & \\
\hline 3 & 0,449 & 0,815 & 0,338 & 0,655 & 0,388 & 1,265 & 0,872 & 1,224 & 0,674 & 0,965 & 0,368 & 1,881 \\
\hline 4 & 0,375 & 0,986 & 0,283 & 0,616 & 0,267 & 1,0 & 0,605 & 1,660 & 0,354 & 1,0 & 0,250 & 1,438 \\
\hline 5 & 323 & 0,690 & 0,372 & 0,461 & 0,294 & 1,899 & 0,323 & 2,411 & 0,449 & 1,035 & 0,271 & 1,552 \\
\hline 6 & 0,418 & 0,565 & 0,308 & 0,852 & 0,265 & 2,539 & 0,562 & 1,267 & 0,470 & 1,399 & 0,246 & 1,715 \\
\hline 7 & 0,395 & 0,791 & - & - & 0,254 & 3,4 & 0,608 & & - & - & 0,284 & 1,588 \\
\hline 8 & & 1,630 & - & - & 0,213 & 3,351 & 0,459 & & - & - & 0,276 & 1,503 \\
\hline 9 & 0,407 & 1,327 & - & - & - & - & 0,537 & 2,509 & - & - & - & - \\
\hline
\end{tabular}

*1.Versuchsjahr 1997: Ernte 1 bis 3; 2.Versuchsjahr 1998: Ernte 4 bis 8; Messwerte [1].

\section{Strontium- und Barium- Verteilung}

Die Verteilung von Strontium und Barium in Kompartimenten von Datura stramonium, Hyoscyamus niger und Atropa belladonna im Verlauf der Vegetationsperiode ist in Abb. 3 dargestellt. 


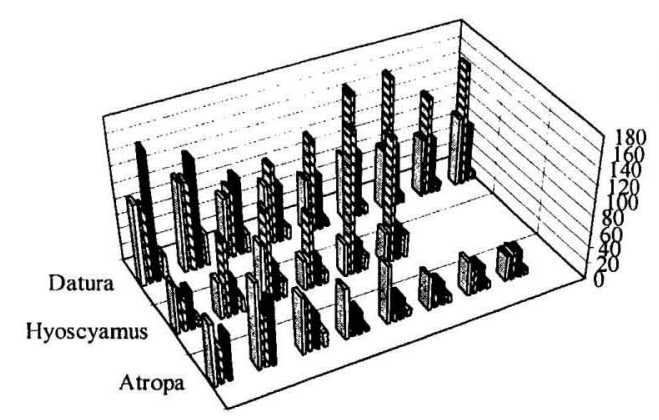

Strontium

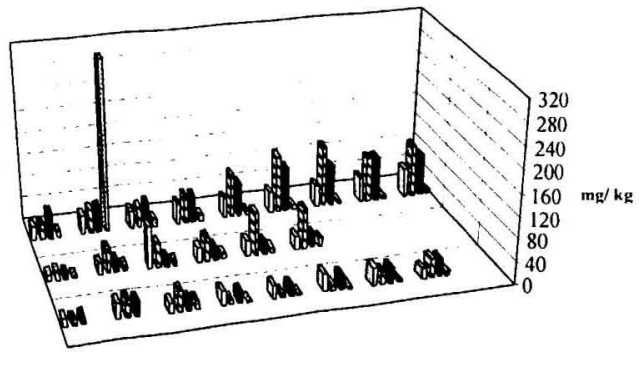

Barium

Abb. 3: Strontium- und Barium-Konzentrationen in den Kompartimenten Wurzeln, Blätter, Stengel und Blüten/Früchte (Reihenfolge innerhalb einer Ernte von links nach rechts) von Datura stramonium ( 9 Ernten) und Hyoscyamus niger (6 Ernten) sowie Atropa belladonna im Verlauf von 2 Jahren ( 3 Ernten im ersten, 5 Ernten im zweiten Jahr); Messwerte [1].

Bei den Proben der ersten drei Ernten von Datura findet man die höchsten Strontium- und Barium-Konzentrationen in den Stengeln - Ausnahme $311 \mathrm{mg} / \mathrm{kg}$ Ba in Blüte/Früchte der 2. Ernte- ab der vierten in den Blättern. Die niedrigsten Konzentrationen, etwa $10 \mathrm{mg} / \mathrm{kg}$, werden in den Blüten/Früchten angetroffen. Bis zu $90 \%$ Strontium ist während der gesamten Vegetationsperiode in den Blättern und Stengeln lokalisiert. Die Barium-Konzentrationen sind bis zu $50 \%$ geringer. Blüten/ Früchte als massenreichste Fraktion enthalten nur ca $10 \%$ des Strontiums und Bariums.

Bei Hyoscyamus weisen die Blätter die höchsten Konzentrationen auf, mit Ausnahme für Strontium bei der ersten Ernte und für Barium bei der dritten Ernte, die der Blüten/Früchte sind stets am niedrigsten.

Atropa zeigt ein anderes Verhalten. Strontium wird, mit Ausnahme der letzten Ernte, vorwiegend in den Wurzeln gefunden. Barium liegt in allen Fraktionen in gleicher Größenordnung vor. Die Transferquotienten sind Tab. 4 zu entnehmen.

Tab. 4: Wurzel/Boden (W/Bo)- und Blätter/Wurzel (Bl/W)-Transferquotienten für Strontium und Barium bei Datura stramonium, Hyoscyamus niger und Atropa belladonna.

\begin{tabular}{cccccc|ccccccc}
\hline \multicolumn{7}{c|}{ Strontium } & \multicolumn{6}{c}{ Barium } \\
\hline Ernte & \multicolumn{2}{c}{$\begin{array}{c}\text { Datura } \\
\text { stramonium }\end{array}$} & \multicolumn{2}{c|}{$\begin{array}{c}\text { Hyoscyamus } \\
\text { niger }\end{array}$} & \multicolumn{2}{c}{$\begin{array}{c}\text { Atropa } \\
\text { belladonna* }\end{array}$} & \multicolumn{2}{c}{$\begin{array}{c}\text { Datura } \\
\text { stramonium }\end{array}$} & \multicolumn{2}{c}{$\begin{array}{c}\text { Hyoscyamus } \\
\text { niger }\end{array}$} & \multicolumn{2}{c}{$\begin{array}{c}\text { Atropa } \\
\text { belladonna* }\end{array}$} \\
& W/Bo & Bl/W & W/Bo & Bl/W & W/Bo & Bl/W & W/Bo & Bl/W & W/Bo & Bl/W & W/Bo & Bl/W \\
\hline 1 & 1,064 & 0,935 & 0,631 & 0,732 & 0,811 & 0,559 & 0,066 & 1,111 & 0,030 & 1,482 & 0,039 & 0,684 \\
2 & 1,187 & 0,974 & 0,487 & 2,034 & 1,126 & 0,609 & 0,094 & 1,280 & 0,055 & 2,178 & 0,076 & 1,090 \\
3 & 0,799 & 1,128 & 0,789 & 1,518 & 0,748 & 0,851 & 0,086 & 1,180 & 0,205 & 0,575 & 0,048 & 2,062 \\
4 & 0,802 & 1,288 & 0,392 & 2,410 & 0,664 & 0,176 & 0,104 & 1,289 & 0,072 & 1,617 & 0,069 & 0,234 \\
5 & 0,721 & 1,676 & 0,455 & 4,184 & 0,768 & 0,189 & 0,096 & 2,232 & 0,074 & 2,846 & 0,066 & 0,335 \\
6 & 0,846 & 1,947 & 0,405 & 3,965 & 0,445 & 0,506 & 0,108 & 2,620 & 0,067 & 2,948 & 0,083 & 0,315 \\
7 & 0,775 & 2,193 & - & - & 0,454 & 0,709 & 0,108 & 2,771 & - & - & 0,085 & 0,720 \\
8 & 0,776 & 1,675 & - & - & 0,348 & 1,085 & 0,126 & 1,767 & - & - & 0,046 & 1,953 \\
9 & 0,909 & 1,745 & - & - & - & - & 0,141 & 1,832 & - & - & - & - \\
\hline
\end{tabular}

*1.Versuchsjahr 1997: Ernte 1 bis 3; 2.Versuchsjahr 1998: Ernte 4 bis 8; Messwerte [1]. 


\section{Diskussion}

Die sechs untersuchten Metalle sind nach ihrem Verhalten gegenüber Datura stramonium und Hyoscyamus niger in drei Gruppen aufzuteilen. Aluminium und Chrom werden in den Wurzeln angereichert. Nur in geringem Umfang findet ein Transport in oberirdische Kompartimente statt.

Kupfer und Zink sind innerhalb der untersuchten Pflanzen gut beweglich. Es fallen relativ hohe Gehalte in den Blüten/Früchten auf, in denen sonst oft die geringsten Metallanteile zu finden sind. Eine mögliche Erklärung besteht darin, dass Kupfer und Zink für Pflanzen essentielle Elemente sind, die in ausreichender Menge in den Samen vorhanden sein müssen, um dem Keimling für seine Entwicklung zur Verfügung zu stehen. Für die anderen untersuchten Elemente besteht offensichtlich eine Transportbarriere zu den Früchten.

Strontium und Barium werden hauptsächlich in den Blättern angereichert; auch ist ein Ansteigen der Konzentrationen über die Vegetationsperiode hinweg zu verzeichnen. Strontium ist dabei am Ende der Vegetationsperiode sogar gegenüber dem Boden angereichert. Auffällig sind die niedrigen Konzentrationen in den Blüten/Früchten im Vergleich zu den übrigen Kompartimenten.

Atropa belladonna zeigt insbesondere im zweiten Versuchsjahr ein anderes Verhalten. $\mathrm{Ob}$ diese Abweichungen pflanzenspezifisch sind - es handelt sich im Gegensatz zu den anderen untersuchten Arten um ein mehrjähriges Gewächs - oder ob sich das Verhalten im zweiten Jahr auf insgesamt veränderte Wachstumsbedingungen zurückführen läßt, kann mit den geringen Datenmengen nicht entschieden werden.

Hinsichtlich der Aufnahme der Metalle aus dem Boden in die Pflanze und der Beweglichkeit innerhalb der Pflanze lassen sich vergleichbare Aussagen machen. Aluminium wird in geringem Umfang aufgenommen, obwohl das höchste Angebot vorliegt. Für die drei Pflanzenarten läßt sich nach den Transferquotienten die Reihenfolge $\mathrm{Al}<\mathrm{Ba}<\mathrm{Cr}<\mathrm{Cu}<\mathrm{Zn}<\mathrm{Sr}$ angeben, in der die Elemente von den Wurzeln aus dem Boden aufgenommen werden. Chrom und Aluminium sind innerhalb der Pflanze recht unbeweglich, dagegen zeigen Zink, Barium und Strontium eine gute Beweglichkeit. Von den drei untersuchten Pflanzenarten vermag Atropa Aluminium, Kupfer und Chrom am leichtesten in die Blätter zu verlagern, für Strontium und Barium ist dies bei Hyoscyamus der Fall.

Allgemein fällt bei den Transferquotienten auf, dass sie sich insbesondere im Jugendstadium der Pflanze von den später ermittelten unterscheiden; offenbar werden in diesem Enwicklungsstadium Elemente verstärkt aus dem Boden in die Wurzeln aufgenommen bzw. in die Blätter umgelagert. 


\section{Experimenteller Teil}

Geräte und Methoden

Die ICP-AES wird als sequentielle Analyse mit dem ICP-5500, Perkin Elmer, durchgeführt. Boden und Pflanzenmaterial werden einem Druckaufschluss in edelstahlummantelten PTFE-Gefäßen, ausgestattet mit Druckfedern und Berstscheiben, unterzogen. Zur Vermeidung externer Kontaminationen finden Probenvorbereitung und Messungen, soweit möglich, in einer Reinstraumanlage statt. Alle Gefäße, Pipetten und Gegenstände, mit denen die Proben in Kontakt kommen, werden vorbehandelt, indem sie mindestens 10 Tage lang in 10 proz. $\mathrm{HNO}_{3}$ eingelegt, kurz vor der Verwendung dreimal mit AquaMilliQ gespült und bei $60^{\circ} \mathrm{C}$ getrocknet werden. Zur Parameteroptimierung und Kalibrierung des Spektrometers wird aus den Einzelelement-Standardlösungen durch entsprechende Verdünnung mit 1proz. $\mathrm{HNO}_{3}$ ein Mehrelementstandard mit Endkonzentrationen von $20 \mathrm{mg} / \mathrm{l} \mathrm{Al}$ und $\mathrm{Ba}, 2 \mathrm{mg} / \mathrm{l}$ $\mathrm{Cu}, 10 \mathrm{mg} / \mathrm{l} \mathrm{Cr}$ und $\mathrm{Zn}$ sowie $5 \mathrm{mg} / \mathrm{l} \mathrm{Sr}$ hergestellt. Zur Bestimmung von Al werden die Probelösungen $1+99$ mit lproz. $\mathrm{HNO}_{3}$ verdünnt. Die Auswertung erfolgt direkt gegen die Bezugslösungen.

Zur Qualitätskontrolle des Druckaufschlusses der Bodenmatrix wird das zertifizierte Referenzmaterial BCR No. 142, light sandy soil [3], und für die Pflanzenmatrix SRM 1573a, tomato leaves, analysiert [4]. Es werden jeweils Doppelbestimmungen durchgeführt.

Zur Probenvorbereitung wurden Keramikmesser (Boker ceramic) und eine Gartenschere mit teflonbeschichteten Schneidflächen verwendet, Pipettierungen erfolgten mit Eppendorf-Pipetten, die Aufbewahrung in $20 \mathrm{ml}$ Polyvials der Firma Zinsser.

Chemikalien und Standards; Analysenbedingungen

Alle verwendeten Chemikalien haben, soweit nicht anders angegeben, den Reinheitsgrad p.a.. Es finden Verwendung: 65proz. $\mathrm{HNO}_{3}, 70$ proz. $\mathrm{HClO}_{4}, 40$ proz. $\mathrm{H}_{2} \mathrm{~F}_{2}$ Merck, AquaMilliQ Waters mit einem Widerstand von $18 \mathrm{MegOhm} \mathrm{x}$ cm sowie $\mathrm{lg} / \mathrm{l}$ Einzelelementstandards Merck von $\mathrm{Cr}, \mathrm{Zn}, \mathrm{Al}, \mathrm{Cu}, \mathrm{Sr}$ und $\mathrm{Ba}$. Die Analysenbedingungen zur ICP-AES sind bereits früher beschrieben [5].

Probenvorbereitung

Der Anbau der Pflanzen erfolgte, nach Arten getrennt, auf Versuchsfeldern im Arzneipflanzengarten des Instituts für Pharmazeutische Biologie und Phytochemie der WWU Münster*. Es handelt sich um Parzellen von $25 \mathrm{~m}$ Länge und 1,5 m Breite. Sie liegen durch je ein $1 \mathrm{~m}$ breites Rasenstück getrennt direkt nebeneinander. Räumliche Nähe und Gleichbehandlung veranlaßten uns, Teilproben der Böden einzelner Felder zu einer Gesamtprobe zu vereinigen. Von Parzellen, auf denen Datura stramonium und Atropa belladonna wachsen sollten, wurden jeweils 10 Teilproben, von den Feldern, die für Hyoscyamus niger vorgesehen waren, je 5 Teilproben genommen. Zur Entnahme der Proben wurde jeweils ein $100 \mathrm{ml}$ Meßzylinder aus Polypropylen mit einer Einstichtiefe von 20 bis $25 \mathrm{~cm}$ senkrecht in den Boden gedrückt und der Inhalt samt außen anhaftenden Boden in einen Polyethylenbeutel übergeführt. Nach manueller Zerkleinerung und Trocknen bei $105^{\circ} \mathrm{C}$ bis zur Gewichtskonstanz wurde der Boden durch ein $2 \mathrm{~mm}$-Kunststoffsieb gestrichen und durch Vierteilen auf etwa $100 \mathrm{~g}$ verjüngt [6], in der Analysenmühle pulverisiert und bis zum Gebrauch in Polyethylen-Schraubgefäßen bei $20^{\circ} \mathrm{C}$ dunkel und trocken gelagert. 
Die Bodenproben wurden Mitte Mai 1997 genommen. Nach Anzucht im Warmbereich des Gewächshauses erfolgte die Aussaat im Frühjahr 1997 (100 Exemplare Datura stramonium, 25 Hyoscyamus niger, 60 Atropa belladonna). Zuvor waren alle Parzellen umgepflügt und mit $100 \mathrm{~g}$ Blaukorn $/ \mathrm{m}^{2}$ gedüngt worden. Anfang August erfolgte eine Nachdüngung mit $200 \mathrm{~g}$ Blaukorn $/ \mathrm{m}^{2}$. Die erste Probennahme von Datura stramonium und Hyoscyamus niger wurde 10 Tage nach dem Auspflanzen ins Freiland vorgenommen. Die weiteren Ernten fanden bis zum Versuchsende jeweils am gleichen Tag im Abstand von 2 Wochen statt. Die ersten Exemplare von Atropa belladonna wurden unmittelbar vor der Aussaat entnommen, die weiteren Probenahmen erfolgten aufgrund der langsameren Entwicklung dieser Pflanze jeweils im Abstand von 4 Wochen. In der Regel wurden 3 Exemplare jeder Art geerntet, wobei jeweils nur solche ausgewählt wurden, die den durchschnittlichen Entwicklungsstand des Versuchsfeldes repräsentierten. Darüber hinaus fanden nur solche Pflanzen Berücksichtigung, die über die Parzelle verstreut wuchsen.

Zur Analyse der Bodenmatrix werden 90 bis $110 \mathrm{mg}$ je Probe, genau gewogen, mit $500 \mu \mathrm{l} 65$ proz. $\mathrm{HNO}_{3}$ sowie $600 \mu \mathrm{l} 40$ proz. $\mathrm{H}_{2} \mathrm{~F}_{2}$ versetzt und bei $170^{\circ} \mathrm{C} 6 \mathrm{~h}$ lang einem Druckaufschluß unterzogen. Die Aufschlußsäuren werden bei $150{ }^{\circ} \mathrm{C}$ unter den üblichen Vorsichtsmaßnahmen bis zur Trockne abgeraucht, mit $3 \mathrm{ml} \mathrm{lproz.} \mathrm{HNO}_{3}$ versetzt und 5 min lang erneut erhitzt. Nach dem Abkühlen wird mit lproz. $\mathrm{HNO}_{3}$ quantitativ in einen $10 \mathrm{ml}$ Meßkolben übergeführt, bis zur Eichmarke aufgefüllt und in Polyvials ${ }^{\otimes}$ bei $20^{\circ} \mathrm{C}$ aufbewahrt. Die Aufschlußbedingungen für die Pflanzenmatrix sind bereits früher beschrieben [5].

\section{Literatur}

- Wir danken Herrn Prof. Dr. A. Nahrstedt für seine Unterstützung.

1 Riße, D. (2000), Diss., Universität Münster.

2 Farago, M. E. in Farago, M. E. (Hrsg.), Plants and the Chemical Elements, 1st Edition, S. 227, Verlag Chemie, Weinheim 1994.

3 Colinet, E., Gonska, H., Griepink, B., Muntau, H. (1983), BCR Information Reference Material BCR No. 142, Commission of the European Communinities/Community Bureau of Reference.

4 National Institute of Standards \& Technology, Certificate of Analysis Standard Reference Material 1573a tomato Leaves, NIST Gaithersburg, MD 20899, USA, 1995.

5. Lahl, H., Riße, D., Unterhalt, B. (2000), Sci. Pharm. 68, 435.

6 Lewandowski, J., Leitschuh, S., Koß, V. (1997), Schadstoffe im Boden, 1. Auflage, Springer Verlag Berlin. 